\title{
Global-density fluctuations in methane clathrate hydrates in externally applied electromagnetic fields
}

Conor J. Waldron and Niall J. English

Citation: The Journal of Chemical Physics 147, 024506 (2017); doi: 10.1063/1.4990029

View online: http://dx.doi.org/10.1063/1.4990029

View Table of Contents: http://aip.scitation.org/toc/jcp/147/2

Published by the American Institute of Physics

\section{Articles you may be interested in}

Perspective: Outstanding theoretical questions in polymer-nanoparticle hybrids

The Journal of Chemical Physics 147, 020901 (2017); 10.1063/1.4990501

Coupled cluster valence bond theory for open-shell systems with application to very long range strong correlation in a polycarbene dimer

The Journal of Chemical Physics 147, 024107 (2017); 10.1063/1.4991797

Unveiling the nature of post-linear response Z-vector method for time-dependent density functional theory The Journal of Chemical Physics 147, 024108 (2017); 10.1063/1.4991561

Native point defects on hydrogen-passivated $4 \mathrm{H}-\mathrm{SiC}(0001)$ surface and the effects on metal adsorptions The Journal of Chemical Physics 147, 024707 (2017); 10.1063/1.4991847

The ${ }^{3} \Pi_{g}$ state of $\mathrm{C}_{2}$ : A pathway to dissociation

The Journal of Chemical Physics 147, 024305 (2017); 10.1063/1.4985882

Benchmark studies of variational, unitary and extended coupled cluster methods

The Journal of Chemical Physics 133, 234102 (2010); 10.1063/1.3520564

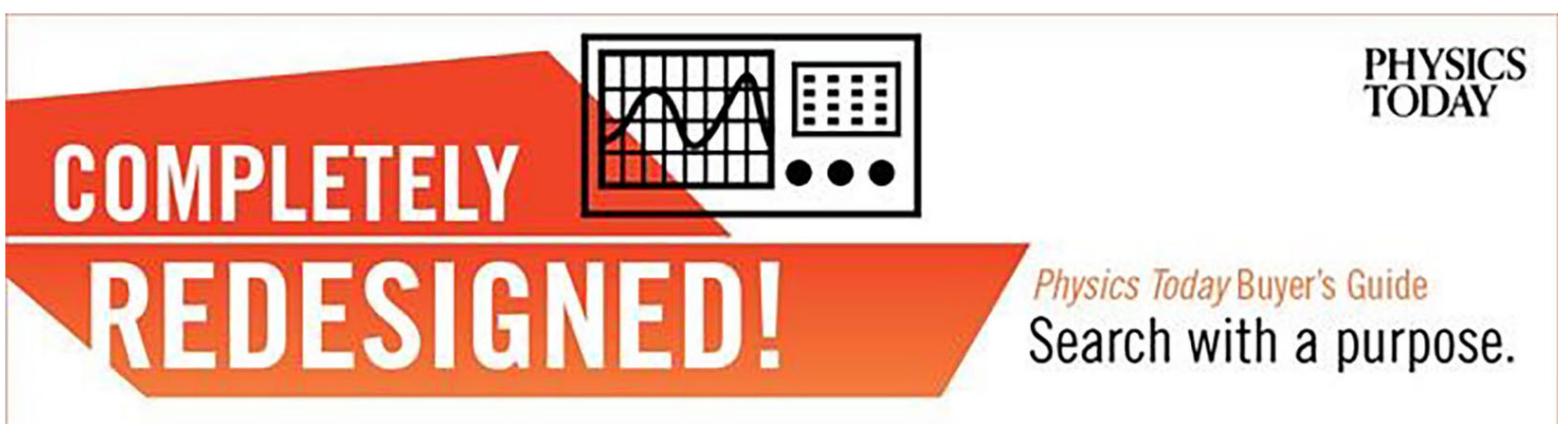




\title{
Global-density fluctuations in methane clathrate hydrates in externally applied electromagnetic fields
}

\author{
Conor J. Waldron a) and Niall J. English ${ }^{\text {b) }}$ \\ School of Chemical and Bioprocess Engineering, University College Dublin, Belfield, Dublin 4, Ireland
}

(Received 8 November 2016; accepted 13 June 2017; published online 14 July 2017)

\begin{abstract}
Non-equilibrium molecular-dynamics simulations of bulk methane clathrate hydrates have been conducted in a range of externally applied electromagnetic $(\mathrm{e} / \mathrm{m})$ fields. Studies into frequencies of system(or "global")-mass-density fluctuations showed that these clathrates have three major modes: the dominant one is attributable to water molecules' librations and occurs at $720 \mathrm{~cm}^{-1}$, regardless of any applied e/m fields. One of the more minor system-density fluctuations arises at $10-12 \mathrm{~cm}^{-1}$ and is caused by the propagation of local-density fluctuations; again, this is independent of e/m fields. The final density fluctuation is caused by e/m fields, and it only becomes apparent for field strengths of $1.2 \mathrm{~V} / \mathrm{nm}$ or higher. The frequency of this mode is always twice the frequency of the applied $\mathrm{e} / \mathrm{m}$ field. It was shown that the main qualitative features of the translational and librational densities of states (DOSs) were unaffected by the application of e/m fields; however, a slight coupling effect was observed, producing a peak in all DOSs at the frequency of the applied field. This study showed that $\mathrm{e} / \mathrm{m}$ fields below a certain intensity threshold do not lead to any marked structural distortion or dissociation effect on pre-existing bulk clathrates, in which the hydrogen-bonding structure of the lattice remains intact. This is verified by system-density and configurational-energy values as well as radial distribution functions. Published by AIP Publishing. [http://dx.doi.org/10.1063/1.4990029]
\end{abstract}

\section{INTRODUCTION}

Gas hydrates are crystalline inclusion compounds, wherein a water host lattice encages small guest atoms or molecules in cavities; the lattice is stabilised by the enclathration of trapped solutes. ${ }^{1,2}$ There are three known common clathrate-hydrate structures: (s)I, II, and H. In sI hydrates, the unit cell is formed from two small $5^{12}$ pentagonal dodecahedral cavities and six slightly larger tetrakaidecahedral $5^{12} 6^{2}$ cages, with 46 water molecules. ${ }^{1,2}$ Methane hydrates are most abundant in situ, occurring in nature in the permafrost and in marine-sediment environments, typically adopting sI structure. Recently, global interest in methane clathrates has been dramatically increasing due to its potential to be a new source of natural gas, motivated in large part by energy and environmental considerations. ${ }^{3-5}$ Naturally, this has led to a wide host of molecular-dynamics (MD) simulations studying methane-clathrate extraction methods, typically from marine milieux. ${ }^{6}$ More generally, molecular simulation has proved to be a rather invaluable tool in revealing much about equilibrium properties of clathrate hydrates, including structure, ${ }^{6-10}$ a "resonant-scattering" picture of phonons and guest motion in cavities, ${ }^{11-13}$ hydrogen-bonding characteristics, ${ }^{14,15}$ and dynamical ${ }^{7-22}$ and energetic properties. ${ }^{10,16}$ Such molecularsimulation studies on dynamical properties have often focused on vibrational properties of the host lattice and on the cavities, with "overlapping" between these acoustic and optic

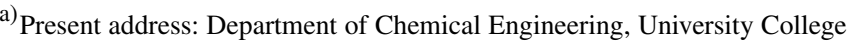
b) London, London, United Kingdom.

b) Author to whom correspondence should be addressed: niall.english@ucd.i.e. Tel.: +353-1-7161646. Fax: +353-1-7161177.
}

modes, often interpreted in the context of resonant scattering. ${ }^{23}$ Such an understanding of equilibrium dynamical properties and phonon scattering offered by molecular dynamics (MD) has indeed led to progress in recent years in enhancing our understanding of thermal-conduction processes in clathrate hydrates, ${ }^{24-28}$ together with "hopping"-mediated guest-diffusion processes of interest of energy- and gas-storage applications. ${ }^{29-33}$

In any event, the application of external electric and electromagnetic $(\mathrm{e} / \mathrm{m})$ fields to gas hydrates is of much technological interest. For instance, in the inhibition of hydrate formation in natural-gas pipelines, aside from the use of either thermodynamic or kinetic inhibitor additives injected into the gas stream, or localised application of higher temperature, ${ }^{1,2}$ external electric and electromagnetic $(\mathrm{e} / \mathrm{m})$ fields may serve to disrupt already-formed hydrates (e.g., as pipeline plugs or possibly in situ "natural" hydrates). ${ }^{1}$ Makogon has commented on the effect of electric fields on hydrate crystals, devising an expression for the shift in thermodynamic equilibrium conditions caused by a static electric or magnetic field, concluding that the electric field intensity would have to be of the order of $10^{7} \mathrm{~V} / \mathrm{m}$ (i.e., $10^{-2} \mathrm{~V} / \mathrm{nm}$ ) to have any significant effect on equilibrium. Makogon also outlined a model for e/m field-induced melting of hydrate deposits from the porous rock of wells. ${ }^{1}$ Rojey has reserved a U.S. patent on a system to inhibit/prevent the formation of clathrate hydrates in pipelines by means of exposure to a field of e/m waves set up by a network of emitters spaced along the length of a pipeline. ${ }^{34}$ Rojey stated briefly that the $\mathrm{e} / \mathrm{m}$ waves, of appropriate frequency and intensity, should prevent or minimise the organisation of water molecules to form a hydrogen-bonded crystalline lattice in a fluid containing an aqueous phase and hydrocarbons, disrupting the possibility 
of hydrate growth. ${ }^{34} \mathrm{e} / \mathrm{m}$ fields would, and should, therefore act as an alternative for gas-pipeline flow-assurance to more established practices of using additives of methanol and other thermodynamic inhibitors. ${ }^{1,2}$ Although e/m-field penetration depths in liquid water are of the order of $\sim 1 \mathrm{~cm}$, in gas, this extends for up to metres; $;{ }^{34}$ this, combined with repeated specular reflection along the pipe walls (in a "zig-zag" fashion), serves to keep the amplitude relatively high over hundreds of metres, which is why Rojey's patent mentions new e/m-field emitters for every few hundred metres of a pipeline. Unfortunately, however, there has been essentially no developmental work of these e/m-field methods for gas-hydrate applications in the past 20 years reported in the open literature, with little industrial $R \& D$ taking place. This relative neglect of this intriguing flow-assurance proposition serves as a motivation to us to study further e/m-field effects on hydrates.

English and MacElroy have carried out non-equilibrium (NE) MD to assess how external e/m fields disrupt the formation of spherical methane hydrate nanocrystals. ${ }^{35}$ They found that the shifting dipolar alignment inside the crystal weakens the structural hydrogen-bonding arrangements, thereby leading to break-up (or inhibition of formation in the first place). Naturally, this is dependent on field intensity and frequency, with intensities of the order of 0.1-0.5 V/nm required to observe tangible results of inhibition within feasible MD simulation times. The "optimal" frequencies for disruption were in the $20-200 \mathrm{GHz}\left(0.67-6.67 \mathrm{~cm}^{-1}\right)$ range (overlapping with the general hydrogen bond lifetimes). This confirms, to some extent, ${ }^{35}$ arguments advanced by Rojey. ${ }^{34}$

Luis et al. subsequently applied static electric fields to bulk methane hydrates, as opposed to spherical nanocrystals, and found that fields greater than $1.5 \mathrm{~V} / \mathrm{nm}$ in intensity led to break up at a pressure of 20 bars and temperature of $248 \mathrm{~K}^{36}$ Luis et al. then performed further "electro-dissociation" of bulk methane hydrates at $260 \mathrm{~K}$ and 80 bars, and also at $285 \mathrm{~K}$ and 400 bars, in static fields of $1-5 \mathrm{~V} / \mathrm{nm}^{37}$

Given that we have recently observed and studied temporal system-density fluctuations in liquid water ${ }^{38}$ and methane hydrates, ${ }^{17}$ in which we established underlying (collective) frequency modes underpinning system-density fluctuations, we now re-focus in the present study on bulk methane hydrates. However, based on Rojey's patent ${ }^{34}$ and English and MacElroy's more fundamental NEMD work ${ }^{35}$ in electromagnetic fields, we are motivated by how external e/m fields may influence or perturb these underlying collective modes. We wish to focus on the athermal, quasi-equilibrium response of the crystalline state to applied e/m fields (albeit with non-equilibrium MD as the tool used). Therefore, we study temporal, systemdensity fluctuations as well as the translational and librational Densities of States (DOSs) of bulk methane hydrates for a range of field frequencies and at intensities up to $2 \mathrm{~V} / \mathrm{nm}$ before the onset of structural distortion (at least on the limited MD time scales used and possible today).

\section{METHODOLOGY}

Equilibrium and non-equilibrium (in-field, vide infra) MD was performed in cubic simulation boxes, for various temperatures and system sizes, under constant-pressure, constant-temperature (NPT) conditions at ambient (1 bar) pressure, using a light coupling to Melchionna's modified form of the Hoover barostat with isotropic cell fluctuations and barostat and thermostat relaxation times of 0.4 and $0.1 \mathrm{ps}$, respectively. ${ }^{40} \mathrm{~A}$ time step of $2 \mathrm{fs}$ was used, with MD conducted under periodic boundary conditions (PBC). The particle-mesh Ewald method was used to handle long-range electrostatic interactions. ${ }^{26,41}$ Normalised autocorrelation functions (ACFs) of the time-derivatives of the system density were computed as follows:

$$
c^{\alpha}(t)=\left\langle\dot{\alpha}_{i}(t) \dot{\alpha}_{i}(0)\right\rangle /\left\langle\dot{\alpha}_{i}(0) \dot{\alpha}_{i}(0)\right\rangle,
$$

where $\alpha$ denotes the density $(m / V$, i.e., the total mass of water and methane molecules per unit of system volume). The use of time-derivatives, $\dot{\alpha}$, in the definition has the advantage of being acutely sensitive to temporal fluctuations (e.g., periodic oscillations or "vibrations") in the density, allowing this to be probed conveniently and straightforwardly via power spectra (Fourier transformation) of their ACFs.

In addition to using Melchionna's modified form of the Hoover barostat, to ascertain the possible effects of barostat relaxation time on temporal system-density fluctuations (i.e., from volume-dilation dynamics), we also applied Gauss's principle of least constraint to both temperature and pressure, so that these are inherent constants of motion. ${ }^{42}$ The Newtonian equations of motion for $\mathbf{r}$ (position) and $\mathbf{p}$ (momentum) of site $i$ in this formulation are altered as follows: ${ }^{42}$

$$
\begin{aligned}
\dot{\mathbf{r}}_{i} & =\dot{\mathbf{p}}_{i} / m_{i}+\dot{\varepsilon} \mathbf{r}_{i}, \\
\dot{V} & =3 V \dot{\varepsilon}, \\
\dot{\mathbf{p}}_{i} & =\mathbf{f}_{i}-\dot{\varepsilon} \mathbf{p}_{i}-\varsigma \mathbf{p}_{i},
\end{aligned}
$$

where the force is $\mathbf{f}_{i}$ and the thermostat's inertia parameter, $\varsigma$, is given by

$$
\boldsymbol{\varsigma}=-\dot{\varepsilon}+\sum_{i=1}^{N} \mathbf{f}_{i} \cdot \mathbf{p}_{i} / \sum_{i=1}^{N} \mathbf{p}_{i}^{2},
$$

whilst the system-volume dilation (or contraction) rate, $\dot{\varepsilon}$, is specified by

$$
\dot{\varepsilon}=\frac{\frac{1}{m} \sum_{i=1}^{N} \sum_{j>i}\left(\mathbf{r}_{i j} \cdot \mathbf{p}_{i j}\right)\left(\phi_{i j}^{\prime \prime}+\phi_{i j}^{\prime} /\left|\mathbf{r}_{i j}\right|\right)}{\sum_{i=1}^{N} \sum_{j>i} \mathbf{r}_{i j}^{2}\left(\phi_{i j}^{\prime \prime}+\phi_{i j}^{\prime}|| \mathbf{r}_{i j} \mid\right)+9 P V} .
$$

In Eqs. (5) and (6), it is understood that the summations run over molecules $i$ and $j$, rather than individual sites, while $\phi_{i j}^{\prime}$ and $\phi_{i j}^{\prime \prime}$ refer, respectively, to the first and second derivatives of the potential. The mass $\mathrm{m}$ refers to that of the molecule, while $\mathrm{P}$ is a pre-defined constant ( $1 \mathrm{bar}$ ) of the motion, but $\mathrm{V}$ varies dynamically [according to Eq. (3)]. Also, $\mathbf{r}_{i j}=\mathbf{r}_{i}-\mathbf{r}_{j}$ and $\mathbf{p}_{i j}$ $=\mathbf{p}_{i}-\mathbf{p}_{j}$, and periodic boundary conditions and holonomic constraints are applied to all position calculations.

Owing to previous simple point charge (SPC) type models being used profitably in local-density fluctuation analysis in liquid water and SPC for system-density-fluctuation analysis in liquid water ${ }^{38}$ and methane hydrates, ${ }^{17}$ we employ here the SPC water model ${ }^{43}$ with an Optimized Potentials for Liquid Simulations - United Atoms (OPLS-UA) methane potential ${ }^{44}$ and Lorentz-Berthelot ${ }^{45}$ combining rules. This performs well 
for hydrate melting-point and free-energy calculations; ${ }^{46}$ further, Luis et al. identified SPC water as being an optimal rigid water molecule for replicating experimentally observed clathrate behaviour around the phase transition. ${ }^{47}$ Autocorrelation functions [of atomic velocity and density, cf. Eq. (1)] were defined for 50 ps using the multiple-time-origin method, ${ }^{45}$ and the ACF amplitude had essentially decayed to zero within 10 ps (and often less). Fourier transformation of ACFs defined in length from 10 to $50 \mathrm{ps}$ showed essentially no variation. Filon's method was used for Fourier transformation, without any smoothing, for greater accuracy. ${ }^{45}$

Given that it was established in Ref. 17 that $2 \times 2 \times 2$ (sI) unit cells per simulation box were satisfactory to investigate (zero-field) system-density fluctuations, we used a $2 \times 2 \times 2$ supercell in the present study. The starting coordinates of the oxygen atoms in the model unit cells of sI hydrates were taken from x-ray diffraction data. ${ }^{48}$ Proton-disordered unit cells were constructed such that water molecules were selected to conform to the Bernal-Fowler rules with vanishingly small total dipole moments. ${ }^{49}$ The theoretical maximum occupancy of 2 and 6 methane molecules in the small and large cages, respectively, was assumed, given typical estimates of $90 \%-95 \%$ occupation experimentally in methane hydrates. ${ }^{1,2}$

In addition to equilibrium, zero-field simulations, we ran non-equilibrium MD (NEMD) for four different electromagnetic-field $\mathrm{rms}$ intensities of $0.8,1.2,1.6$, and $2 \mathrm{~V} / \mathrm{nm}$ (given that the bulk-hydrate electro-dissociation threshold is probably marginally larger in e/m fields than $\sim 1.5$ $\mathrm{V} / \mathrm{nm}$ for static fields) $)^{36,37}$ and for three different field frequencies of $1.67,16.67$, and $33.33 \mathrm{~cm}^{-1}$ (i.e., 50, 500, and $1000 \mathrm{GHz}$, respectively) - a total of twelve different e/mfield conditions. These disparate frequencies were chosen to provide a broad spread over the microwave to near-infrared region, and a similar range was used for NEMD of liquid water in terms of gauging e/m-field effects on, inter alia, self-diffusivity. ${ }^{50}$ The e/m fields were applied by perturbing Newton's equation ${ }^{39,50,51}$

$$
m_{i} \ddot{\mathbf{r}}_{i}=\mathbf{f}_{i}+q_{i} \mathbf{E}(t)+q_{i} \mathbf{v}_{i} \times \mathbf{B}(t) .
$$

Here, $q_{i}$ is the charge and $\mathbf{f}_{i}$ is the force on the site $i$. The $\mathrm{e} / \mathrm{m}$ field was taken to be uniform and plane-polarised in the $z$ direction, i.e., the electric component was taken to act in this direction. The magnetic component was taken to act in the $y$ direction, and the plane of polarisation was then the $x-z$ plane, with the field intensities related by $E(t) / B(t)=c$, where $c$ is the speed of light, i.e.,

$$
\begin{aligned}
& \mathbf{E}(t)=E_{\max } \cos (\omega t)(0 \mathbf{i}+0 \mathbf{j}+1 \mathbf{k}) \text { and } \\
& \mathbf{B}(t)=B_{\max } \cos (\omega t)(0 \mathbf{i}+1 \mathbf{j}+0 \mathbf{k})
\end{aligned}
$$

and $E_{\max }=\sqrt{2} E_{\mathrm{rms}}$. Given the non-reversibility of the propagator in Eq. (7) for magnetic fields, simulations were repeated with a $1 \mathrm{fs}$ time step in the case of the $1.2 \mathrm{~V} / \mathrm{nm}$ and $16.67 \mathrm{~cm}^{-1}$ condition, yielding the same density and velocity spectra as those with the 2 fs-step simulations. After zero-field relaxation for $0.5 \mathrm{~ns}$, each production simulation was performed for $0.5 \mathrm{~ns}$ at $250 \mathrm{~K}$ and $60 \mathrm{~atm}$.

It should be noted that the e/m-field intensities used in this study correspond to torques on water molecules' dipoles of typically less than $\sim 10 \%$ of those present in condensed phases of water, i.e., the applied fields are roughly an order of magnitude less than intrinsic electric fields present in condensedwater phases. ${ }^{51-53}$ In the supplementary material, a wider discussion has been added on the relationship between external electric fields used typically in experiments and the physical approximations involved. In brief, the applied-field intensities used here would lead to ionisation, but the rigid nature of the water models prevents this; such high field intensities are necessary to observe a tangible signal-to-noise ratio for nonthermal effects over accessible simulation time scales of the order of a nanosecond.

Dynamic guest-host (methane-water) energy transfer may be investigated via calculation of velocity autocorrelation functions (VACFs; $m=n$ ), i.e.,

$$
Z(t)=\left\langle\mathbf{v}_{\mathrm{m}}(0) \cdot \mathbf{v}_{\mathrm{n}}(t)\right\rangle /\left\langle\mathbf{v}_{\mathrm{m}}(0) \cdot \mathbf{v}_{\mathrm{n}}(0)\right\rangle .
$$

Normalised velocity autocorrelation functions (VACFs) measure the degree of significance of coupling of atomic motions. These were computed for the oxygen and hydrogen atoms in water and for the carbon atoms of the UA methane molecules. Cosine Fourier transformation (power spectra) of the VACFs reveals the densities of states; ${ }^{16-20,23,54}$ the oxygen atoms in water dominate the translational motion of the lattice, whilst the hydrogen atoms reflect librational motion. ${ }^{17}$ The translational vibrations of the encaged methane molecules may be studied in the power spectrum of the methane carbon atoms (centre-of-mass). ${ }^{16-19}$ In any event, we may also study e/mfield effects on the DOS, as has been done for VACF-spectra in chiral liquids and their racemic mixtures,${ }^{55}$ as well as for liquid water ${ }^{56}$ and water adsorbed at metal-oxide surfaces. ${ }^{57}$

\section{RESULTS AND DISCUSSION}

For liquid water, the average intrinsic electric-field intensities were found to range from about 15 to $25 \mathrm{~V} / \mathrm{nm}$. The probability distributions of the electric field strengths are shown in Fig. 1 for liquid water at 260-400 K. Here, the Coulombic forces were evaluated on each charge site (excluding the force from the external field) from Ewald-propagated MD, and $\mathbf{E}$ at each such site $i$ is defined as $\mathbf{f}_{\mathrm{i}} / \mathrm{q}_{\mathrm{i}}$, with its magnitude then taken. The intrinsic electric-field intensities in the bulk hydrate phase were very similar to this range, justifying the earlier

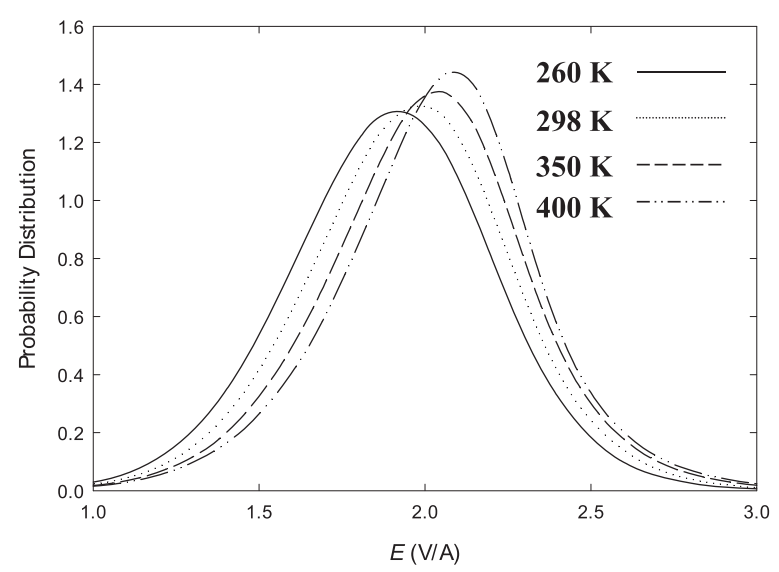

FIG. 1. Probability distribution of intrinsic electric-field intensities in liquid water at various temperatures. 
observation of the torques on water molecules' dipoles of typically less than $\sim 10 \%$ of those present in condensed phases of water.

The average density and configurational energy of the clathrate system were largely unaffected by e/m fields, as seen in Table I. This indicates that no dissociation occurred, confirmed by applying Báez-Clancy geometric hydraterecognition criteria. ${ }^{58}$ In any event, inducing hydrate dissociation is not a goal of this study.

At first glance, this may appear surprising because English and MacElroy have shown for TIP4P-FQ water models that $\mathrm{e} / \mathrm{m}$ fields of up to $\sim 8 \mathrm{~cm}^{-1}(\sim 240 \mathrm{GHz})$ with rms intensities of $1 \mathrm{~V} / \mathrm{nm}$ or higher can break apart methane-hydrate nano-crystallites (of $\sim 2.5 \mathrm{~nm}$ in diameter) and inhibit their formation. ${ }^{35}$ However, as discussed earlier, a clathrate nanocrystal is, ipsofacto, an incomplete structure lacking the crystal structure of the bulk, with only a certain number of hydrogen bonds stabilising a limited number of complete clathrate cages. In comparison, in the bulk crystal (as handled by PBC), there is an infinite number of cages in all directions and this cage structure creates mutually reinforcing hydrogen bonds, creating added stability for the clathrates. Therefore, it is not surprising that an e/m field of up to $\sim 1-2 \mathrm{~V} / \mathrm{nm}_{\text {rms }}$ can induce dissociation in weaker nano-crystallite systems, but not in the more stable bulk clathrates (at least on time scales of up to 0.5 ns). In contrast, static fields are oriented in one direction, with an unchanging intensity, allowing easier rupture of stabilising hydrogen bonds in hydrates for $1.5 \mathrm{~V} / \mathrm{nm}$ and above..$^{36,37}$

At this point, it is instructive to compare, qualitatively, the fundamentally differing effects of static and e/m fields. In the case of liquid water, static fields causing a permanent (partial) re-alignment of water molecules' dipoles, ${ }^{59-61}$ whereas e/m fields instead lead to (partial) continuous rotation of water molecules, due to the cosine-varying torque on their dipoles [cf. Eq. (3) and Refs. 39, 51, and 52]. As mentioned above, in the case of hydrates, static fields above a certain threshold intensity (e.g., $\sim 1.5 \mathrm{~V} / \mathrm{nm}$, at least for nanosecond
NEMD-simulation periods) $)^{36,37}$ induce clathrate dissociation because the greater water-dipole alignment leads to distortion of the clathrate structure, as the water molecules are rotated out of their original crystallographic positions, and the cage/lattice-stabilising hydrogen bonds are ruptured. In comparison, the (partial) rotation induced relatively readily by $\mathrm{e} / \mathrm{m}$ fields in liquid-like water (or dipolar liquid) environments ensures dissipation of the mechanical work done on the dipoles by the field's torques into re-orientational motion, primarily, albeit with some extent of roto-translational coupling ${ }^{62}$ (e.g., enhancement of translational self-diffusivity). ${ }^{51,63,64}$ However, in the case of hydrates, whether in bulk-crystal or nanocrystallite form, below the intensity threshold needed for $\mathrm{e} / \mathrm{m}$-induced electro-dissociation (which will vary naturally with external-field frequency), ${ }^{35}$ the dissipation of torquemediated work is targeted primarily into librational (rotationoscillation $^{18,19}$ ) modes (and vibrational modes, if a flexible model is used).$^{50}$ In this case, then, there is primarily an "enhanced rattling" effect on the hydrogen-bonding network, rather than outright re-orientational motion leading to rupture of stabilising bonds. Therefore, with the application of such "sub-threshold" e/m fields, there are no evident structural changes or adjustments in the strength of the hydrogen-bonding network. This is in accord with Table I.

In order to further quantify any structural changes, or lack of thereof, in the clathrate system, radial distribution functions (RDFs) were obtained. It was found that none of the $\mathrm{e} / \mathrm{m}$ fields studied, regardless of intensity or frequency, had any effect on any RDF. This is demonstrated by Figs. 2(a) and 2(b) which are representative of all RDFs observed from every simulation conducted. Figure 2(a) shows the (waterwater) oxygen-oxygen RDF for all intensities run at $1.67 \mathrm{~cm}^{-1}$ $(50 \mathrm{GHz})$, whilst Fig. 2(b) depicts this for all frequencies run at $2 \mathrm{~V} / \mathrm{nm}_{\mathrm{rms}}$; the corresponding RDF under a zero-field condition serves as a reference.

The RDFs for the zero-field simulation, and indeed of all the in-field simulations, show the ordered nature of a

TABLE I. Average values and standard deviations of density in $\mathrm{kg} / \mathrm{m}^{3}$ and configurational-energy values (per water) in $\mathrm{kJ} / \mathrm{mol}$.

\begin{tabular}{lccccc}
\hline \hline $\begin{array}{l}\text { rms } \\
\text { intensity }\end{array}$ & Frequency & Density & $\begin{array}{c}\text { Standard deviations } \\
\text { of density }\end{array}$ & $\begin{array}{c}\text { Average configurational } \\
\text { energy (per water) }\end{array}$ & $\begin{array}{c}\text { Standard deviations } \\
\text { of configurational } \\
\text { energy (per water) }\end{array}$ \\
\hline$(\mathrm{V} / \mathrm{nm})$ & $\left(\mathrm{cm}^{-1}\right)$ & $\mathrm{kg} / \mathrm{m}^{3}$ & $\mathrm{~kg} / \mathrm{m}^{3}$ & $\mathrm{~kJ} / \mathrm{mol}$ & $\mathrm{kJ} / \mathrm{mol}$ \\
Zero-field & Zero-field & 932.1 & 6.3 & -53.538 & 0.439 \\
0.8 & 1.67 & 931.9 & 5.9 & -53.492 & 0.343 \\
0.8 & 16.67 & 931.3 & 6.7 & -53.480 & 0.485 \\
0.8 & 33.33 & 931.7 & 7.7 & -53.538 & 0.607 \\
1.2 & 1.67 & 931.4 & 6.3 & -53.421 & 0.418 \\
1.2 & 16.67 & 931.2 & 6.6 & -53.413 & 0.460 \\
1.2 & 33.33 & 931.3 & 7.3 & -53.409 & 0.561 \\
1.6 & 1.67 & 930.9 & 5.2 & -53.292 & 0.255 \\
1.6 & 16.67 & 930.8 & 5.2 & -53.321 & 0.267 \\
1.6 & 33.33 & 930.6 & 8.0 & -53.304 & 0.649 \\
2.0 & 1.67 & 929.5 & 5.6 & -53.417 & 0.372 \\
2.0 & 16.67 & 930.7 & 5.0 & -53.174 & 0.259 \\
2.0 & 33.33 & 930.0 & 10.7 & -53.141 & 0.962 \\
\hline \hline
\end{tabular}




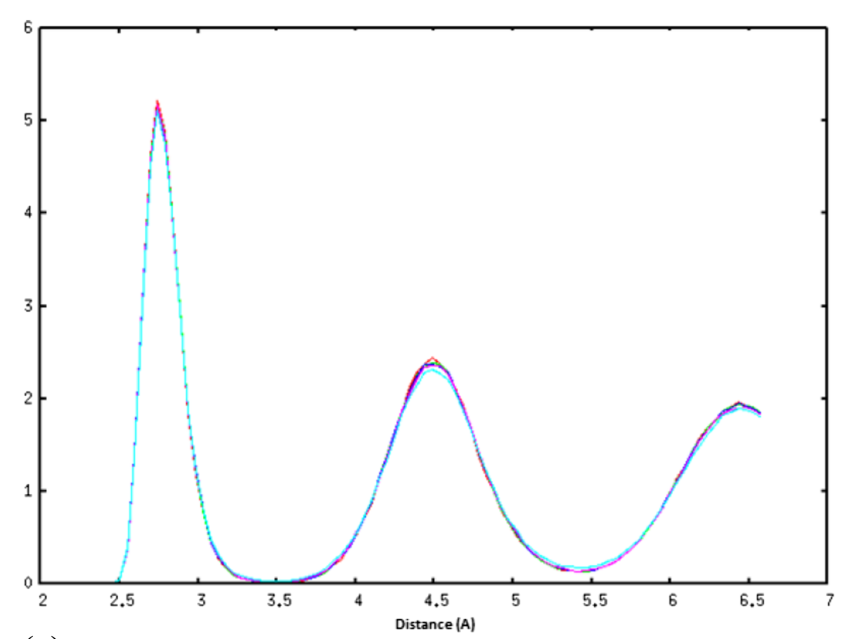

(a)

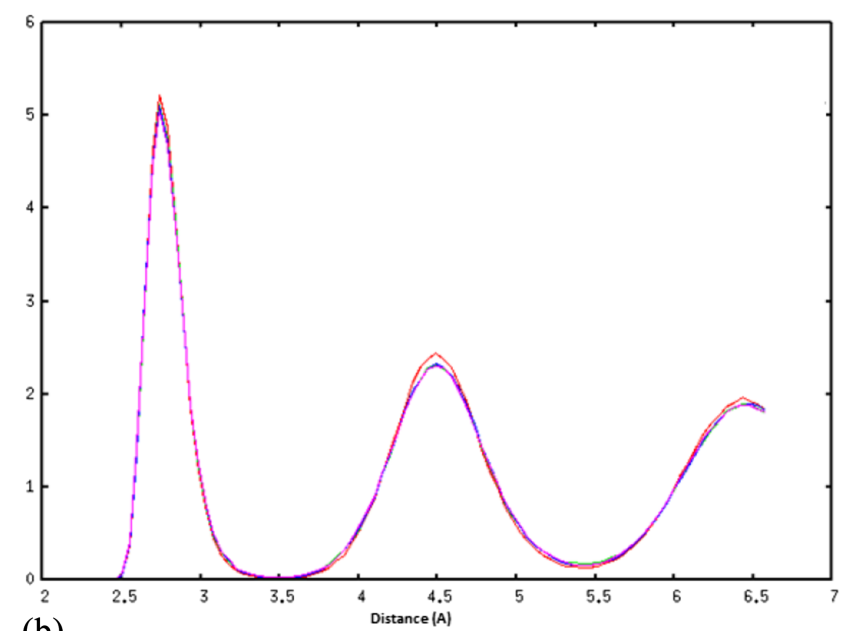

(b)

FIG. 2. (a) Oxygen-oxygen RDF of methane clathrate under zero-field conditions (red), and $16.67 \mathrm{~cm}^{-1} \mathrm{e} / \mathrm{m}$ fields of $0.8 \mathrm{~V} / \mathrm{nm}_{\mathrm{rms}}$ (green), $1.2 \mathrm{~V} / \mathrm{nm}_{\mathrm{rms}}$ (blue), $1.6 \mathrm{~V} / \mathrm{nm}_{\mathrm{rms}}$ (purple), and $2 \mathrm{~V} / \mathrm{nm}_{\mathrm{rms}}$ (turquoise). (b) Oxygen-oxygen RDF of methane clathrate under zero-field conditions (red) and $2 \mathrm{~V} / \mathrm{nm}_{\mathrm{rms}}$ $\mathrm{e} / \mathrm{m}$ fields with frequencies of $1.67 \mathrm{~cm}^{-1}$ (green), $16.67 \mathrm{~cm}^{-1}$ (blue), and $33.33 \mathrm{~cm}^{-1}$ (purple).

clathrate hydrate as the RDF consists of sharp definite peaks, characteristic of the cage network and similar to comparable RDFs in Refs. 17 and 54. The observation that the application of e/m fields of up to $2 \mathrm{~V} / \mathrm{nm}_{\text {rms }}$ of any frequency in the range studied has no effect on the RDF is in agreement with the earlier density and configurational-energy results (cf. Table I).

The RDF and density results are in good accord with previously published results. ${ }^{10,16,17,19,23,54}$ In particular, the RDFs have the same sharp features at the right radial distances that essentially match for a variety of different potential models. In addition, the density results are in good agreement with typical experimental values. ${ }^{1,2}$ Although not the most sophisticated potentials, the currently used ones do capture the melting point and basic thermodynamic and structural properties quite well. ${ }^{17,46}$

A study was conducted on the frequency of the fluctuations in system mass density (i.e., total system mass divided by total system volume) and configurational energy, to identify the microscopic phenomena creating these fluctuations and to observe how these phenomena are affected by $\mathrm{e} / \mathrm{m}$ fields. The Fourier transform of the ACF of the derivative of both density and configurational energy was calculated to identify the dominant fluctuation frequencies [cf. Eq. (1), where $\alpha$ is system mass-density or configurational energy]. Three major density fluctuations were observed and Table II shows the wavenumber of each of these fluctuations for each simulation run. The dominant fluctuation occurred at $\sim 720 \mathrm{~cm}^{-1}$ for all simulations and it was attributed to the librational motion of water molecules. ${ }^{17-19}$ The other two density fluctuations were of much smaller magnitude and of lower frequency. One of these fluctuations occurred at $10-12 \mathrm{~cm}^{-1}$ for all simulations, while the last density fluctuation was only observed for higher-intensity e/m fields and it had a frequency of twice the applied e/m field.

As stated earlier, the dominant frequency occurred at approximately $720 \mathrm{~cm}^{-1}$ for every set of conditions studied and is attributed to librations of water molecules. Libration is a repetitive restricted oscillation in rotational motion. In the case of liquid water, molecules' librations occur because hydrogen bonding prevents the water molecules from rotating freely (as in the gas phase), driven by their thermal energy. Therefore, in the hydrate, instead of making full $360^{\circ}$ rotations, water molecules experience librations where they "wiggle" or rotate back and forth in small angles, and it is this repetitive rotation and straining against hydrogen bonds that causes system-density fluctuations, albeit at the high frequency of individual molecular librations. Here, it was found that the application of $\mathrm{e} / \mathrm{m}$ fields did not influence the magnitude of frequency of libration: this is not unexpected because it has already been shown that e/m fields affect hydrogen-bond kinetics markedly but do not cause any changes in the strength of the hydrogen-bonding network. Since librations are controlled by the strength of the restrictive hydrogen bonds on a water molecule (e.g., in a hydrate lattice), ${ }^{18}$ it is therefore logical that $\mathrm{e} / \mathrm{m}$ fields (at least in the lower-frequency microwave and infrared range) will not influence librations.

For in-field NEMD, a low-frequency system-density temporal fluctuation is observed for higher-strength fields, in terms of the overall system density of the system (as opposed

TABLE II. Frequency of librational, e/m-field, and system-density fluctuations (in $\mathrm{cm}^{-1}$ ).

\begin{tabular}{lccccc}
\hline \hline \multicolumn{2}{c}{ Simulations } & & \multicolumn{3}{c}{ Density fluctuations } \\
\cline { 1 - 2 } \cline { 5 - 6 } $\begin{array}{l}\text { rms intensity } \\
(\text { V/nm })\end{array}$ & $\begin{array}{c}\text { Frequency } \\
\left(\mathrm{cm}^{-1}\right)\end{array}$ & $\begin{array}{c}\text { Libration peak } \\
\left(\mathrm{cm}^{-1}\right)\end{array}$ & $\begin{array}{c}\text { e/m peak } \\
\left(\mathrm{cm}^{-1}\right)\end{array}$ & $\begin{array}{c}\text { Local peak } \\
\left(\mathrm{cm}^{-1}\right)\end{array}$ \\
\hline Zero-field & Zero-field & & 720 & N/A & 12 \\
0.8 & 1.67 & & 725 & Not observed & 12 \\
0.8 & 16.67 & & 725 & Not observed & 12 \\
0.8 & 33.33 & & 725 & Not observed & 12 \\
1.2 & 1.67 & & 724 & Not observed & 12 \\
1.2 & 16.67 & & 725 & 33 & 12 \\
1.2 & 33.33 & & 725 & Not observed & 12 \\
1.6 & 1.67 & & 722 & 3 & 12 \\
1.6 & 16.67 & & 722 & 33 & 12 \\
1.6 & 33.33 & & 722 & 66 & 12 \\
2.0 & 1.67 & & 715 & 3 & 12 \\
2.0 & 16.67 & & 722 & 33 & 12 \\
2.0 & 33.33 & 720 & 66 & 12 \\
\hline \hline
\end{tabular}


to any local measure of density). The observed density fluctuation's frequency is twice that of the applied e/m field, and it is first observed at $1.2 \mathrm{~V} / \mathrm{nm}_{\text {rms }}$; however, it is only observed consistently for field strengths of $1.6 \mathrm{~V} / \mathrm{nm}_{\text {rms }}$ or higher. Figure 3 shows the Fourier transform of the densityderivative ACF for the $2 \mathrm{~V} / \mathrm{nm}_{\text {rms }}$ simulations where e/m-field fluctuations are clearly visible as peaks. This fluctuation, even when observed, is far smaller and less significant than the dominant libration peak. It is thought that this fluctuation is caused by $\mathrm{e} / \mathrm{m}$ fields forcing water molecules to rotate (only partially), without rupturing the lattice's stabilising hydrogen bonds, causing the system density to swell and contract periodically. The average peak-to-trough amplitude of the volume dilations corresponded to $\sim 0.72 \%, 0.93 \%, 1.52 \%$, and $1.74 \%$ for the $0.8,1.2,1.6$, and $2 \mathrm{~V} / \mathrm{nm}$ fields, respectively, with those in the $1.67 \mathrm{~cm}^{-1}$ fields displaying a slightly larger $(\sim 10 \%)$ amplitude than the higher intensity ones (due to a longer field period in which the system volume could respond). In the $0.8 \mathrm{~V} / \mathrm{nm}$ case, this was found to be almost 4 times higher than the zero-field density-fluctuation magnitude. The study of the spectra of the ACF of the configurational energy's derivative [cf. Eq. (3) with $\alpha$ as configurational energy] shows that the configurational energy also fluctuates with a frequency of twice the applied $\mathrm{e} / \mathrm{m}$ field, even at intensities lower than the threshold intensity to observe the density fluctuation. The "double-frequency" nature of e/m-induced modes arising from the system-density and configurational-energy's derivative-ACFs is conjectured to originate from the pairwise molecular interactions defining these fundamental quantities of energy and density: the $\mathrm{e} / \mathrm{m}$-field's oscillating torque acting on the water molecules' dipole vectors sets up an "echo" effect of an opposing torque from the surrounding respective molecules (as a consequence of neighbouring molecules' force response via Newton's third law); this serves to double the de facto $(\mathrm{e} / \mathrm{m})$ torque-oscillation (or frequency) experienced by each molecule. In any event, given that configurational energy is an average over individual molecules, it is, in a sense, a single-molecule property, whilst system mass density is an inherently collective property; based on this, it is not surprising to see the e/m-field vibrational signature at lower applied-field intensities in the single-molecule

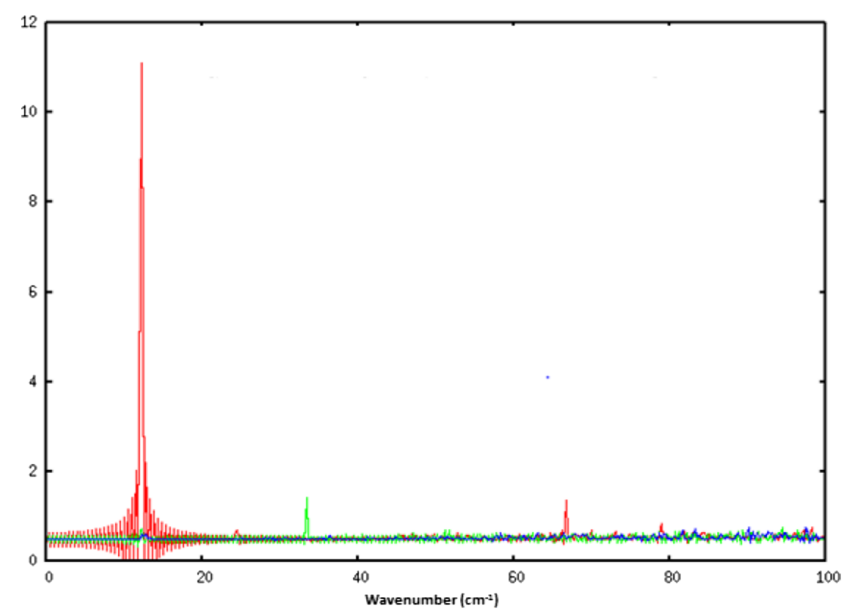

FIG. 3. Low-frequency section of the Fourier transform of the densityderivative ACF of methane clathrates exposed to $2 \mathrm{~V} / \mathrm{nm}_{\mathrm{rms}}$ fields with frequencies of $1.67 \mathrm{~cm}^{-1}$ (blue), $16.67 \mathrm{~cm}^{-1}$ (green), and $33.33 \mathrm{~cm}^{-1}$ (red).

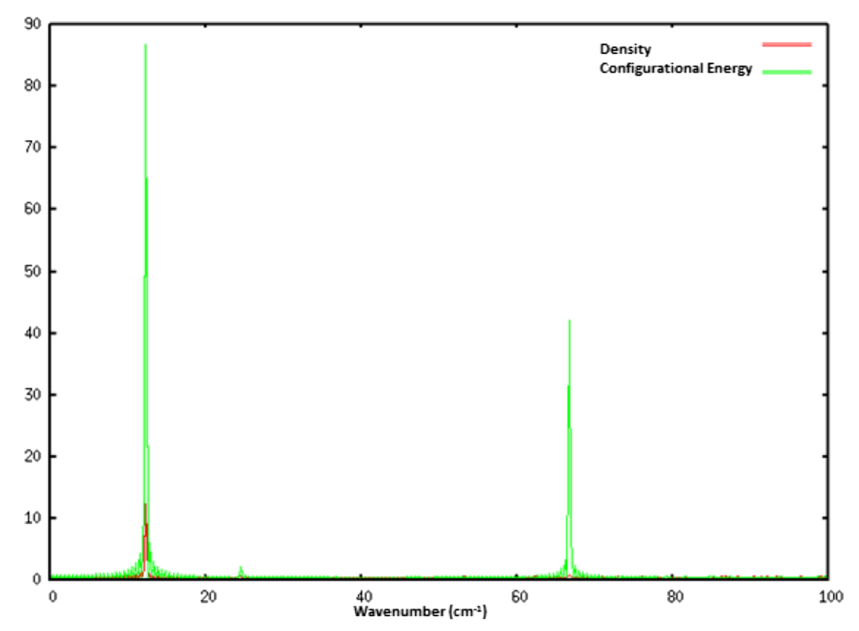

FIG. 4. Low-frequency section of the Fourier transform of the densityderivative $\mathrm{ACF}$ (red) and configurational-energy ACF (green) of methane clathrates under $1.6 \mathrm{~V} / \mathrm{nm}_{\mathrm{rms}}, 33.33 \mathrm{~cm}^{-1} \mathrm{e} / \mathrm{m}$ fields.

property (i.e., ACF of configurational-energy derivatives). An example is shown in Fig. 4. It is postulated that this threshold intensity of 1.2-1.6 V/nm ${ }_{\text {rms }}$ to observe a density fluctuation of twice the $\mathrm{e} / \mathrm{m}$ field frequency is due to the hydrogen-bonding in clathrates being resilient to the studied applied fields.

The clathrate system is shown to exhibit a density fluctuation with a frequency of $10-12 \mathrm{~cm}^{-1}$. This fluctuation is observed for all simulations and is independent of any variable studied, as demonstrated in Fig. 3. This fluctuation is also observed in the ACF of configurational energy as shown in Fig. 4. This frequency is found to be produced by localdensity fluctuations ${ }^{65}$ resulting in small system-density fluctuations. ${ }^{17,38}$ This conclusion is drawn because the frequency of this peak, $12 \mathrm{~cm}^{-1}$, corresponds to a period of $\sim 3 \mathrm{ps}$ which is close to the known lifetime of a local-density fluctuation of less than 4 ps. ${ }^{17,38}$ Also, in NPT dynamics involving Gauss's principle of least constraint [cf. Eqs. (2)-(6)], which is independent of the reservoirs' thermal- and pressure-dissipation inertia, the system-density fluctuations were observed to be essentially identical.

The host-lattice translational and vibrational DOSs, i.e., VACF spectra of oxygen and hydrogen atoms, respectively, and those of methane, were calculated for each simulation run in order to identify the influence of $\mathrm{e} / \mathrm{m}$ fields on the rattling modes of a bulk clathrate system. For methane centre-of-mass vibrations, the three peaks are consistent with the almost elliptical shape of the large cages and spherical shape of the small ones. The zero-field DOS, shown in Fig. 5, is in agreement with those reported in a previous study of a range of different potential models in zero-field conditions. ${ }^{17}$ The host translational DOS shows the expected peaks at $\sim 70$ and $240 \mathrm{~cm}^{-1}$ which are caused by acoustic phonons and transverse optical modes, respectively. ${ }^{7,8,11,12,17,54}$ The lattice's vibrational DOS exhibits a broad peak within the previously quoted range of $400-1400 \mathrm{~cm}^{-1,7,8,17,54}$ this peak is caused by librations of water molecules.

It was found that $\mathrm{e} / \mathrm{m}$ fields of any intensity or of frequency up to $16.67 \mathrm{~cm}^{-1}$ have essentially no effect on the main features of the VACF spectra for oxygen, hydrogen, or methane; this is demonstrated by Figs. 6(a) and 6(b), which 


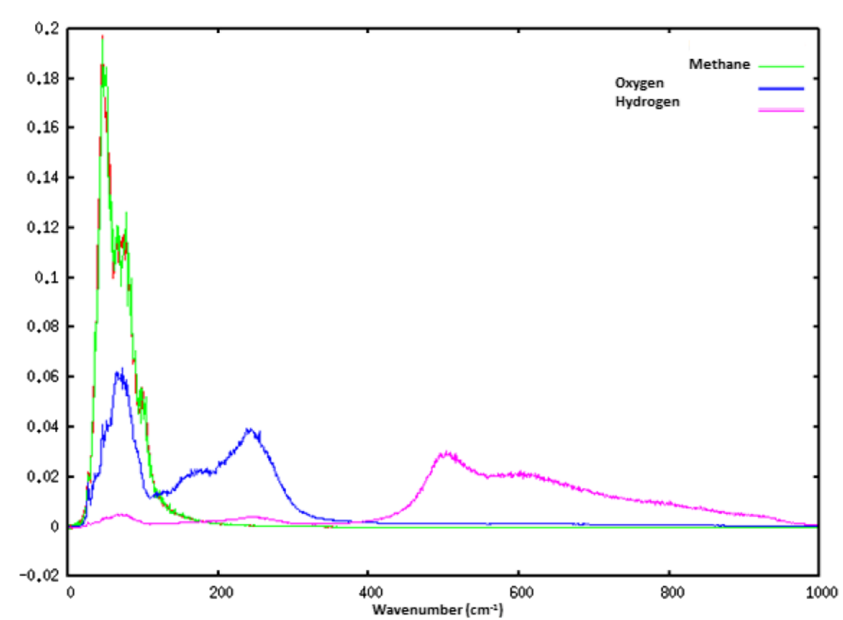

FIG. 5. Fourier transform of the VACF for methane (green) and for the host lattice's water oxygen atoms (blue) and hydrogen atoms (purple) for a zerofield simulation of methane clathrate at $250 \mathrm{~K}$ and $60 \mathrm{~atm}$.

show the host's translational DOS under $16.67 \mathrm{~cm}^{-1}$ fields of differing intensities and under a range of different frequency $2 \mathrm{~V} / \mathrm{nm}_{\mathrm{rms}}$ e/m fields. Both Figs. 6(a) and 6(b) contain a zerofield simulation for comparison and are representative of all the DOSs for oxygen, hydrogen, and methane under any e/m field. However, in Fig. 6(b), there is an e/m field coupling for $33.33 \mathrm{~cm}^{-1}$ and $2 \mathrm{~V} / \mathrm{nm}_{\text {rms }}$ case (by way of a clear peak at $33.33 \mathrm{~cm}^{-1}$ ), which was essentially absent at lower intensities. This $1 \mathrm{THz}$ e/m field oscillates comparatively rapidly (in the infrared region) and is relatively intense (at $\sim 10 \%$ of the intrinsic electric field), and therefore yields an oscillatingtorque-induced mode in the DOS of the translational lattice, given that it is nearer in frequency to the host lattice's acoustic modes vis-à-vis the lower-intensity e/m fields applied here. The observation that the main peaks of the DOSs were independent of e/m fields (below $2 \mathrm{~V} / \mathrm{nm}_{\mathrm{rms}}$, at any rate) was somewhat expected because, as discussed above, the e/m fields in this intensity range have been shown to only affect the kinetics of hydrogen bonding markedly, ${ }^{50,51,61}$ instead of leading to even moderate structural changes or clathrate dissolution. The hydrate lattice, especially in the bulk state, does not exhibit any hydrogen-bond rupture over $0.5 \mathrm{~ns}$, although this might well be expected under longer simulation durations, especially in the nanoscale-crystallite state, as shown in Ref. 35. Over longer (experimental) time scales, this e/m-response would be of interest to the macroscopic, experimental dielectric-response measurements of Bertie et al. ${ }^{66,67}$ which established that water molecules rotate about half as rapidly as those in ice, even though the hydrogen bonds are similar in strength to those in ice. ${ }^{68}$ Rick and Freeman ${ }^{69}$ elucidated the proton-disorderpropagation mechanisms explaining this dielectric response, using hybrid Monte Carlo and MD, but this would not be possible with deterministic NEMD in the presence of e/m fields. Substantially longer simulations, approaching milliseconds in relatively intense e/m fields, such as those studied here, would be needed to probe e/m-field effects on enhancing rotational motion in host-lattice water molecules, thereby accelerating the underlying proton-disorder-propagation mechanisms and leading to more rapid dielectric relaxation (and a corresponding lower dielectric constant). ${ }^{66,67}$

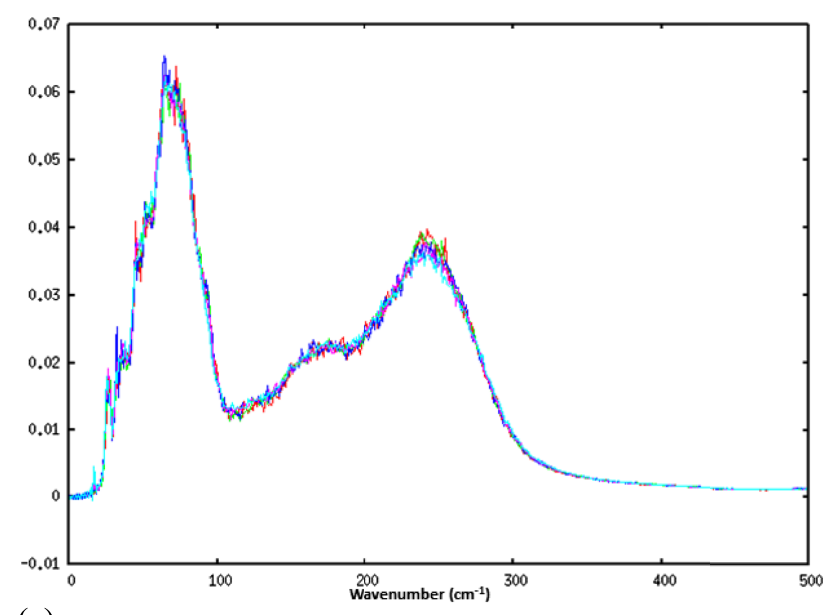

(a)

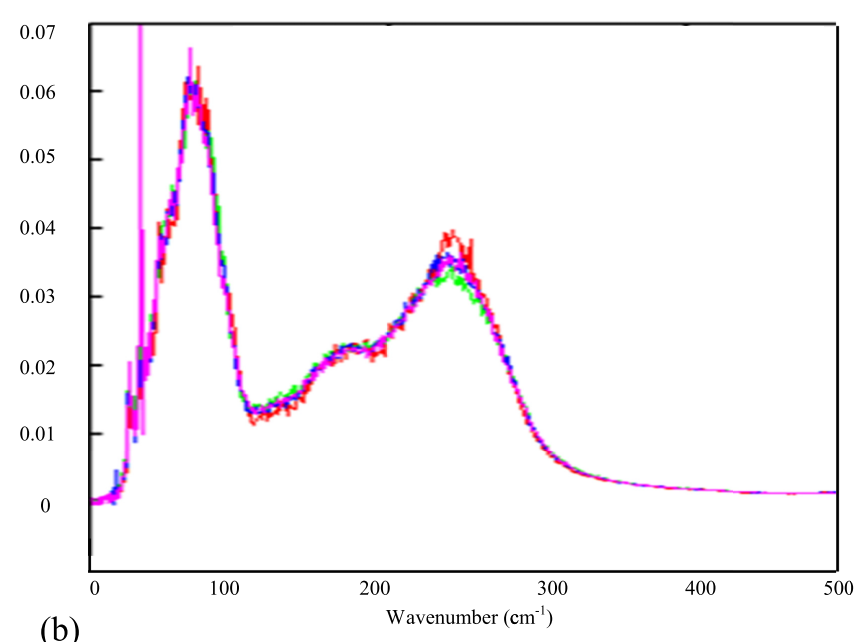

FIG. 6. (a) Fourier transform of the velocity ACF for oxygen in no field (red) and in a range of $16.67 \mathrm{~cm}^{-1} \mathrm{e} / \mathrm{m}$ fields with intensities of $0.8 \mathrm{~V} / \mathrm{nm}_{\mathrm{rms}}$ (green), $1.2 \mathrm{~V} / \mathrm{nm}_{\text {rms }}$ (blue), $1.6 \mathrm{~V} / \mathrm{nm}_{\text {rms }}$ (purple), and $2 \mathrm{~V} / \mathrm{nm}_{\mathrm{rms}}$ (turquoise). (b) Fourier transform of the velocity ACF for oxygen in a zero field (red) and in a range of $2 \mathrm{~V} / \mathrm{nm}_{\mathrm{rms}} \mathrm{e} / \mathrm{m}$ field frequencies, $1.67 \mathrm{~cm}^{-1}$ (green), $16.67 \mathrm{~cm}^{-1}$ (blue), and $33.33 \mathrm{~cm}^{-1}$ (purple).

However, although e/m fields do not affect the dominant peaks of the DOS, they have been shown to cause the formation of small peaks of the same frequency as the applied $\mathrm{e} / \mathrm{m}$ field. These e/m peaks are observed for all of the DOSs, which includes the DOS of oxygen, hydrogen, and methane in both large and small cages. The magnitude of the e/m peak is found to increase with the intensity of the applied field and the peaks are only easily visible at intensities of $1.6 \mathrm{~V} / \mathrm{nm}_{\mathrm{rms}}$ or greater. Furthermore the magnitude of the peak increases with the frequency of the applied field, as shown in Fig. 7. This e/m peak is especially interesting, as it occurs at the same frequency as that of the applied e/m field instead of at twice the frequency as was observed for density and configurational-energy fluctuations. A similar coupling effect between $\mathrm{e} / \mathrm{m}$ fields and dipole alignment has been observed in previous studies of liquid water. ${ }^{38}$ In the present case, for hydrates, the coupling of the VACF and the e/m field can be explained by both oxygen and hydrogen being charged species: this means that they will exhibit direct velocity responses to the e/m field, and, therefore, the VACF-spectrum peak is expected at the applied field frequency. Although the methane 


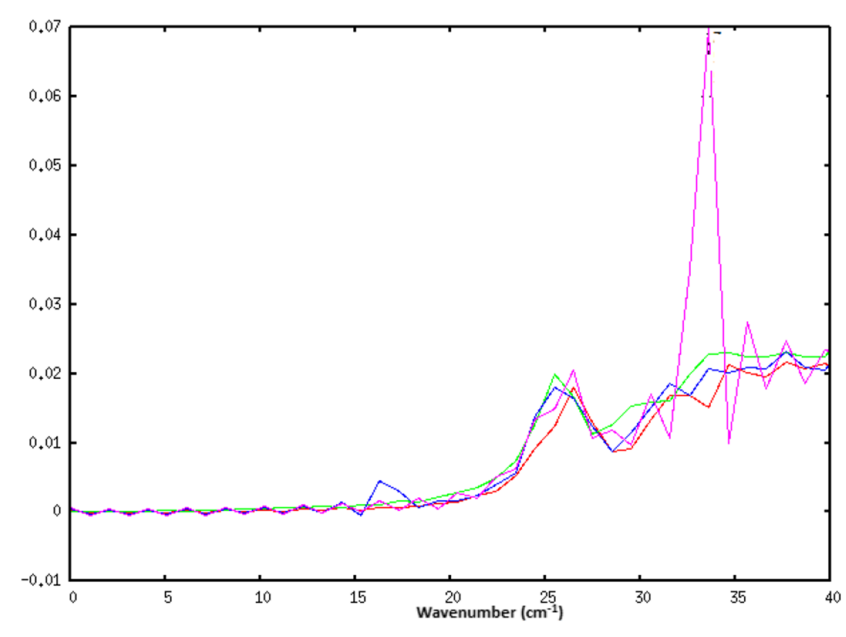

FIG. 7. Translational DOS of host lattice (i.e., Fourier transform of the wateroxygen VACF) under a zero field (red) and in a range of $2 \mathrm{~V} / \mathrm{nm}_{\mathrm{rms}} \mathrm{e} / \mathrm{m}$ field frequencies, $1.67 \mathrm{~cm}^{-1}$ (green), $16.67 \mathrm{~cm}^{-1}$ (blue), and $33.33 \mathrm{~cm}^{-1}$ (purple).

molecules do not respond directly to the e/m field, owing to their symmetric partial-charge structure and no dipole, it is thought that the corresponding peak in methane VACFspectra is caused by the field-induced oxygen and hydrogen positional fluctuations being transmitted across to methane through collisions between methane and the surrounding water cages; this, of course, is reinforced by the concept of resonant scattering. ${ }^{23}$

\section{CONCLUSIONS}

Through NEMD in external e/m fields, to probe athermal effects in the quasi-equilibrium state, it has been shown that microwave and infrared $\mathrm{e} / \mathrm{m}$ fields in the intensity range studied do not bring about appreciable structural changes to bulk methane clathrates, despite the fact that e/m fields can inhibit clathrate formation. ${ }^{34,35}$ It is important to note that electrodissociation was not a goal of the present study. The lack of structural change is because when e/m fields cause water molecules to rotate, it affects primarily librational modes (not in terms of frequency per se, but rather in terms of mechanicalwork leakage into those modes); it is only in the case of outright rupture of the lattice by more intense fields that the rate of rearrangements of the hydrogen bonds would be accelerated (as happens in liquid water at all e/m-field intensities)..$^{50,51,61}$ The lack of field-induced structural effects was demonstrated by no major changes in density, configurational energy, RDFs, or DOS.

It was observed that the clathrate density fluctuated with three separate frequencies, the dominant frequency being caused by the librational motion of water molecules, which is independent of e/m fields. The second frequency of density fluctuation was found to be twice the frequency of any applied e/m field, although this fluctuation was only observed above a threshold intensity of $1.2 \mathrm{~V} / \mathrm{nm}_{\text {rms }}$. This density fluctuation is produced by water molecules being forced into angular positions of maximum and minimum repulsion from their neighbours attainable without rupture of stabilising hydrogen bonds, which also produces fluctuations of the same frequency in configurational energy. The final frequency of density fluctuation $\left(\sim 10-12 \mathrm{~cm}^{-1}\right)$ is attributed to local-density fluctuations. ${ }^{17,38}$ The main features of the DOS of oxygen, hydrogen, and methane were unaffected by e/m fields, as e/m fields do not induce any structural changes to the clathrate system. However, it was found that the e/m fields did have a small direct coupling effect on velocities, as manifested in the DOS where a small peak in the VACF-spectra is produced at the same frequency as that of the applied $\mathrm{e} / \mathrm{m}$ field. This coupling effect is caused because the partially charged atoms experience a direct velocity response to the $\mathrm{e} / \mathrm{m}$ field (by field-induced torque on the water dipole).

\section{SUPPLEMENTARY MATERIAL}

See supplementary material for the description of electricfield intensities typically used in simulations and how it is compared with both the experiment and the currently used external-field intensities.

${ }^{1}$ Y. F. Makogon, Hydrates of Hydrocarbons (PennWell Books, Tulsa, Oklahoma, 1997).

${ }^{2}$ E. D. Sloan and C. A. Koh, Clathrate Hydrates of Natural Gases, 3rd ed. (CRC Press, Taylor \& Francis, USA, 2007).

${ }^{3}$ G. J. MacDonald, Annu. Rev. Energy 15, 53-83 (1990).

${ }^{4}$ K. A. Kvenvolden, Chem. Geol. 71, 41-51 (1988).

${ }^{5}$ P. G. Brewer, F. M. Orr, G. Friederich, K. A. Kvenvolden, and D. L. Orange, Energy Fuels 12, 183-188 (1998).

${ }^{6}$ Y.-T. Tung, L.-J. Chen, Y.-P. Chen, and S.-T. Lin, J. Phys. Chem. B 115, 15295-15302 (2011).

${ }^{7}$ J. S. Tse, M. L. Klein, and I. R. McDonald, J. Phys. Chem. 87, 4198-4203 (1983).

${ }^{8}$ J. S. Tse, M. L. Klein, and I. R. McDonald, J. Chem. Phys. 81, 6146-6153 (1984).

${ }^{9}$ J. S. Tse, W. R. McKinnon, and M. Marchi, J. Phys. Chem. 91, 4188-4193 (1987).

${ }^{10}$ A. A. Chialvo, M. Houssa, and P. T. Cummings, J. Phys. Chem. B 106, 442-451 (2002).

${ }^{11}$ J. S. Tse, V. P. Shpakov, V. P. Murashov, and V. R. Belosludov, J. Chem. Phys. 107, 9271-9274 (1997).

${ }^{12}$ J. S. Tse, V. P. Shpakov, V. R. Belosludov, F. Trouw, Y. P. Handa, and W. Press, Europhys. Lett. 54, 354-360 (2001).

${ }^{13}$ J. S. Tse, Ann. N. Y. Acad. Sci. 715, 187-206 (1994).

${ }^{14}$ R. Susilo, S. Alavi, I. L. Moudrakovski, P. Englezos, and J. A. Ripmeester, ChemPhysChem 10, 824 (2009).

${ }^{15}$ S. Alavi, K. Udachin, and J. A. Ripmeester, Chem. - Eur. J. 16, 1017 (2010).

${ }^{16}$ P. D. Gorman, N. J. English, and J. M. D. MacElroy, Phys. Chem. Chem. Phys. 13, 19780 (2011).

${ }^{17}$ C. J. Waldron, M. Lauricella, and N. J. English, Fluid Phase Equilib. 413, 235 (2016).

${ }^{18}$ C. J. Burnham and N. J. English, J. Chem. Phys. 144, 051101 (2016).

${ }^{19}$ C. J. Burnham and N. J. English, J. Chem. Phys. 144, 164503 (2016).

${ }^{20}$ N. J. English and J. S. Tse, J. Phys. Chem. A 115, 6226 (2011).

${ }^{21}$ M. Hiratsuka, R. Ohmura, A. K. Sum, and K. Yasuoka, J. Chem. Phys. 136, 044508 (2012).

${ }^{22}$ M. Hiratsuka, R. Ohmura, A. K. Sum, and K. Yasuoka, J. Chem. Phys. 137, 144306 (2012).

${ }^{23}$ N. J. English and J. M. D. MacElroy, Chem. Eng. Sci. 121, 133 (2015).

${ }^{24}$ N. J. English and J. S. Tse, Phys. Rev. Lett. 103, 015901 (2009).

${ }^{25}$ N. J. English, R. Gallagher, and J. S. Tse, Phys. Rev. B 82, 092201 (2010).

${ }^{26}$ N. J. English, Mol. Phys. 106, 1887 (2008).

${ }^{27}$ N. J. English and J. S. Tse, Comput. Mater. Sci. 49, S176 (2010).

${ }^{28}$ N. J. English, P. D. Gorman, and J. M. D. MacElroy, J. Chem. Phys. 136, 044501 (2012).

${ }^{29}$ T. J. Frankcombe and G.-J. Kroes, J. Phys. Chem. C 111, 13044-13052 (2007).

${ }^{30}$ P. D. Gorman, N. J. English, and J. M. D. MacElroy, J. Chem. Phys. 136, 044506 (2012).

${ }^{31}$ H. Cao, N. J. English, and J. M. D. MacElroy, J. Chem. Phys. 138, 094507 (2013). 
${ }^{32}$ T. T. Trinh, M. H. Waage, T. S. van Erpa, and S. Kjelstrup, Phys. Chem. Chem. Phys. 17, 13808 (2015).

${ }^{33}$ C. J. Burnham and N. J. English, J. Phys. Chem. C 120, 16561-16567 (2016).

${ }^{34}$ A. Rojey, "Process and system using an electromagnetic wave to prevent the formation of hydrates," U.S. patent no. 5625178 (29 April 1997).

${ }^{35}$ N. J. English and J. M. D. MacElroy, J. Chem. Phys. 120, 10247 (2004).

${ }^{36}$ D. P. Luis, J. López-Lemus, and M. Mayorga, Mol. Simul. 36, 461-467 (2010).

${ }^{37}$ D. P. Luis, E. C. Herrera-Hernández, and H. Saint-Martin, J. Chem. Phys. 143, 204503 (2015).

${ }^{38}$ C. J. Waldron and N. J. English, Chem. Phys. Lett. 649, 119-122 (2016).

${ }^{39}$ N. J. English and C. J. Waldron, Phys. Chem. Chem. Phys. 17, 12407-12440 (2015).

${ }^{40}$ S. Melchionna, G. Ciccotti, and B. L. Holian, Mol. Phys. 78, 533 (1993).

${ }^{41}$ U. Essmann, L. Perera, M. L. Berkowitz, T. Darden, H. Lee, and L. G. Pedersen, J. Chem. Phys. 103, 8577 (1995).

${ }^{42}$ D. J. Evans and G. P. Morriss, Statistical Mechanics of Nonequilibrium Liquids (Academic Press, London, 1990).

${ }^{43}$ H. J. C. Berendsen, J. P. M. Postma, W. F. van Gunsteren, and J. Hermans, in Intermolecular Forces, edited by B. Pullman (Reidel, Dordrecht, 1981), p. 331.

${ }^{44}$ W. L. Jorgensen, J. D. Madura, and C. J. Swenson, J. Am. Chem. Soc. 106, 6638-6646 (1984).

${ }^{45}$ M. P. Allen and D. J. Tildesley, Computer Simulation of Liquids (Clarendon, Oxford, 1987).

${ }^{46}$ R. E. Westacott and P. M. Rodger, Chem. Phys. Lett. 262, 47 (1996).

${ }^{47}$ D. P. Luis, J. López-Lemus, and L. Romero-Salazar, Mol. Simul. 36, 35-40 (2009).

${ }^{48}$ R. K. McMullan and G. A. Jeffrey, J. Chem. Phys. 42, 2725 (1965).
${ }^{49}$ A. Rahman and F. H. Stillinger, J. Chem. Phys. 57, 4009 (1972).

${ }^{50}$ N. J. English and J. M. D. MacElroy, J. Chem. Phys. 119, 11806 (2003).

${ }^{51}$ N. J. English and J. M. D. MacElroy, J. Chem. Phys. 118, 1589-1592 (2003).

${ }^{52}$ Z. Futera and N. J. English, J. Phys. Chem. C 120, 19603-19612 (2016).

${ }^{53}$ R. S. Kavathekar, P. Dev, N. J. English, and J. M. D. MacElroy, Mol. Phys. 109, 1649 (2011).

${ }^{54}$ N. J. English and J. M. D. MacElroy, J. Comput. Chem. 24, 1569-1581 (2003).

${ }^{55}$ N. J. English, P. G. Kusalik, and S. A. Woods, J. Chem. Phys. 136, 094508 (2012).

${ }^{56}$ R. Reale, N. J. English, P. Marracino, M. Liberti, and F. Apollonio, Chem. Phys. Lett. 582, 60-65 (2013).

${ }^{57}$ Z. Futera and N. J. English, "Oscillating electric-field effects on adsorbedwater at rutile- and anatase- $\mathrm{TiO}_{2}$ surfaces," J. Chem. Phys. 145, 204706 (2016).

${ }^{58}$ L. A. Báez and P. Clancy, Ann. N.Y. Acad. Sci. 715, 177-186 (1994).

${ }^{59}$ N. J. English, G. Y. Solomentsev, and P. O'Brien, J. Chem. Phys. 131, 035106 (2009).

${ }^{60}$ N. J. English, D. A. Mooney, and S. O’Brien, Mol. Phys. 109, 625-638 (2011).

${ }^{61}$ R. Reale, N. J. English, P. Marracino, M. Liberti, and F. Apollonio, Mol. Phys. 112, 1870-1878 (2014).

${ }^{62}$ M. W. Evans, Phys. Rev. Lett. 50, 371 (1983).

${ }^{63}$ N. J. English and J. M. D. MacElroy, Mol. Phys. 100, 3753 (2002).

${ }^{64}$ N. J. English, Mol. Phys. 104, 243 (2006).

${ }^{65}$ N. J. English and J. S. Tse, Phys. Rev. Lett. 106, 037801 (2011).

${ }^{66}$ J. E. Bertie, F. E. Bates, and D. K. Hendricksen, Can. J. Chem. Phys. 53, 71 (1975).

${ }^{67}$ J. E. Bertie and S. M. Jacobs, J. Chem. Phys. 69, 4105 (1978).

${ }^{68}$ S. N. Chakraborty and N. J. English, J. Chem. Phys. 143, 154504 (2015).

${ }^{69}$ S. W. Rick and D. L. Freeman, J. Chem. Phys. 132, 054509 (2010). 